Review

\title{
Building a global psychological science through research in the Pacific Island nation of Fiji: a systematic review of the literature
}

\author{
Annie E. Crookes ${ }^{1} \cdot$ Meg A. Warren ${ }^{2}$ \\ Received: 14 January 2022 / Accepted: 28 January 2022 \\ Published online: 04 March 2022 \\ (c) The Author(s) 2022 OPEN
}

\begin{abstract}
There is increasing globalisation of psychological science through cross-cultural research, international conferences, and funding initiatives. However, it is important to understand the nature of this globalisation in a more nuanced way and for research to include both etic (universal comparisons) and emic (distinctive cultural understanding) approaches and to incorporate the needs and expertise of the Indigenous populations being studied. The present systematic review aimed to identify the psychological research undertaken in the culturally diverse Pacific Island Country of Fiji and explore how this has added to the general knowledge base in psychological science. Furthermore, the review aimed to use the Fiji research literature to evaluate the extent of globalisation in psychology from an etic, emic and Indigenous psychology perspective. A total of 131 peer-reviewed publications were identified on electronic databases of which $80 \%$ reported primary research studies in some form. The literature suggests a growing interest in Pacific-inclusive research that has already added substantially to psychological understanding in many core areas. However, the literature continues to be dominated by etic, cross cultural studies driven by western research interests and universal measures. The discussion suggests more needs to be done to incorporate emic approaches and Indigenous methods as well as consider applied themes and research questions that would better serve the local communities alongside scientific knowledge.
\end{abstract}

Keywords Systematic review · Fiji · Pacific Island Countries · Psychological science · Indigenous psychology

\section{Introduction}

The lack of diversity and global representation in psychological science has been well-documented [1-6]. In a recent article, Tindle [6] noted this cultural bias towards western populations continues to be systemic from authorship of psychology publications and membership of editorial boards, to bias in the samples and findings of accepted studies. This not only impacts the scientific knowledge of human psychology but also how it is applied in culturally diverse therapeutic settings [7-9] and community psychology movements [10]. To some extent, mainstream psychological journals have started to actively promote a more global psychology. For example, Webster et al. [11] report a 7.6\% increase per decade (data from 1978 to 2008) in the number of first authors from outside the US published in APA journals as well as a 4.8\% increase per decade of international members on APA journal editorial boards. Apicella et al. [12] summarises some of the key studies in areas of psychology and cognitive science that have led to a more global understanding of core psychological theories in the past 10 years. Berry [4] also discusses active growth in global representation through international psychological associations (e.g., The International Union of Psychological Science; The International Association of Applied

Annie E. Crookes, annie.crookes@usp.ac.fj|'School of Law and Social Sciences, University of South Pacific, Laucala Campus, Suva, Fiji. ${ }^{2}$ College of Business and Economics, Western Washington University, Bellingham, USA.

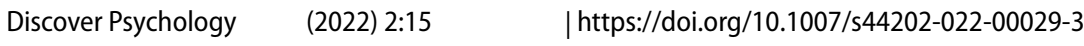


Psychology) which had previously been largely American-led despite purporting to address an international perspective. However, both Apicella et al. [12] and Thalmayer et al. [13] argue that little overall change has happened in the published research sampling which still only represents $11 \%$ of the global population from predominantly English-speaking western nations [13]. In addition, there have been attempts to address the barriers caused by economic and academic resource limitations in non-western countries through globalised funding and academic networking initiatives [14].

However, it is important to understand the nature of this globalisation in a more nuanced way. Adair [1] noted two ways to study psychological constructs and build knowledge within a diverse range of cultural contexts: through cross-cultural comparisons of psychological phenomena; and by employing an Indigenous, focal approach to understanding ethnic and cultural groups in both western and non-western nations. This distinction is also reflected in the discussion of etic and emic approaches to understanding psychological phenomena across cultures [15]. This debate, particularly applied to the field of personality, has questioned whether the purpose of psychological research should be on identifying a set of universal constructs in different cultural contexts or seeking to understand the nature of independent Indigenous psychologies $[16,17]$. Of course, both approaches are necessary to building a psychological science that reliably portrays and predicts human behaviour $[18,19]$. Nevertheless, in many ways it is only through proactive engagement with the more complex and idiosyncratic emic-Indigenous research that psychology can become objectively global and shift away from the many forms of western bias. As such, discussions of globalisation in psychology should also assess the relative portion of etic and emic studies from international populations to evaluate the true breadth of globalisation efforts.

The Indigenous psychology approach both incorporates and extends the emic perspective on psychological research. That is, beyond scientific goals, Indigenous psychology utilises psychological science to address the social issues and needs within the Indigenous population [20]. This includes the extent to which research includes or is driven by researchers from within the population asking research questions developed from their own understanding of the cultural context [10, 20-24]. In addition, the aims and outcomes of the research should be more applied and politically informed than may be seen in other scientific research, in order to positively and directly impact the study population. For example, McNamara and Naepi [10] argued that incorporating an Indigenous approach (as opposed to cross-cultural comparisons) within psychological teaching and research not only informs the field itself but is also a key part of decolonising and empowering the Indigenous populations. As such, an exploration of globalisation from the perspective of Indigenous psychology should take into consideration the extent to which the research sampling from these communities and populations impacts them in a direct and overt way.

\subsection{Research setting: the Pacific Island Countries}

As interest in the globalisation of psychological research grows, one region that offers significant potential to explore a wide range of cultural, economic, and social contexts is the Pacific Island Countries (PICs). The PICs are a cooperative of 14 independent island nations classified as developing countries, alongside the developed nations of Australia and New Zealand. Although these nations have relatively small Indigenous populations, they cover a geographic area equating to $15 \%$ of the Earth's surface (https://www.worldbank.org/en/country/pacificislands) and have significant diversity in language, geography, industry, belief systems and political history. This points to the rich potential they may have to enhance understanding of sociocultural influence on psychological constructs. While many of the PICs do not yet have the infrastructure to accommodate significant academic research, the nation of Fiji is more economically developed and with established academic institutions. Fiji is categorised as a middle-income country based in the Melanesian area of the South Pacific and includes 300 islands. The majority of the 900,000 population live on the two main islands of Viti Levu (hosting the capital city Suva) and Vanua Levu. Fiji was granted independence by the British in 1970, becoming a democratic nation, though it has experienced several political coups (most recently in 2006). There are two primary ethnic groups: the Indigenous iTaukei Fijians who are of Melanesian and Polynesian heritage, and the Indo-Fijians who were brought to Fiji as indentured labourers and professional migrants during colonisation.

Despite a rich diversity in socio-cultural factors that may add value to the globalisation of psychological science, the relatively small island populations within the Pacific Island region (including Fiji) have tended to be only indirectly included within cross-cultural psychological research. For example, some studies make reference to a broader 'Asia Pacific' region that is variously defined as countries including Japan, China, South Korea, Philippines, Indonesia and India [25-27]. Other studies separate countries in Asia from the region of 'Oceania' that tends to be represented predominantly by New Zealand and Australia [1,28]. Therefore, this review will highlight the value of cultural investigations from smaller populations such as Fiji from their own unique cultural perspective rather than regional generalisations. This review in some ways follows on from the global overview by Apicella et al. [12] that provided examples of individual studies from 
populations across the globe that have impacted theoretical psychology. In the present review, we aim to continue this by exploring in more depth how research specifically from the Pacific Island nation of Fiji has enhanced the knowledge of how different cultural and religious landscapes interact with environmental landscapes to create social and individual behaviours.

In addition, the present review also aims to understand how research in PICs such as Fiji represent the opportunity to develop a potentially richer, emic approach to psychological science that benefits from an Indigenous perspective. For example, there are numerous mental health and social issues known to be prominent in Fiji such as poverty, non-communicable diseases, gender-based and intimate partner violence, sexual abuse, and suicide. These need to be investigated from a localised psychological perspective not only to help policy and prevention within pacific communities but also to better understand psychosocial risk and protective factors that may be relevant to the global audience. By considering the emic and Indigenous focused research that has been undertaken in Fiji, this review can also add to ongoing developments in Indigenous psychology as an independent research field.

In summary, there has been a long-established drive for greater globalisation of psychological science as well as a recent interest in specifically indigenous psychology. Fiji as a middle income small island country in the pacific with a rich and diverse cultural population/historic influence provides a good opportunity to better understand the extent of globalisation of psychological science. The research literature investigating psychological themes in a Fiji-based population will be reviewed and summarised. First the review will identify how Fijian psychological studies have added to an understanding of culture and environment on human behaviour and cognitive processes. Second the review will summarise the studies in Fiji that have specifically related to applied themes and add to a global understanding of risk and protective factors in health and wellbeing behaviours. Furthermore, the literature will be evaluated for the number of studies that take etic versus emic approaches as well as studies which align with the Indigenous psychology field. Finally, the review will consider how psychological research has developed in Fiji and whether this reflects sustainable capacity building for a globalised psychological science within Fiji and the Pacific.

\section{Method}

\subsection{Literature search}

Peer-reviewed articles published before December 2020 were searched within various electronic database using keywords "psychology" AND "Fiji", "cognition" AND "Fiji", "mind" AND “Fiji", "mental health" AND "Fiji", "wellbeing" AND "Fiji", "psych*" AND "Fiji", "psych*" AND "South Pacific", "psych*" AND " Pacific Islands". An initial search yielded a total of 1165 hits. Of these 905 had accessible abstracts or full text for review. Screening revealed that most were either duplicates or did not report psychological research or critical discussions based in Fiji, and were therefore, eliminated (see Fig. 1). The final number of individual articles, from peer reviewed publications, with accessible summary or full text and relating to psychology research or practice in Fiji (individually or as part of a multi-country project) was 131.

\subsection{Coding}

The identified publications were first categorised by the lead author from analysis of the paper aims and methods (the sample must have been taken from a location within Fiji and include Native-born Fijians). The categories utilised included: (1) year (2) article type (empirical or non-empirical) (3) methods for empirical research articles (primary or secondary data; quantitative or qualitative methods) (4) sample characteristics (age, gender, location) and (5) a coding for cultural approach and purpose was developed based on etic, emic and Indigenous psychology aims. These codes are defined in Table 1 below.

The identified publications were then grouped by the predominant psychological theme as it relates to psychological fields such as social, cognition, developmental, organizational, clinical, or counseling fields. Where an individual topic within a field appeared in a number of articles this was used as a separate classification for the purposes of the literature discussion. The final theme groups used were: child/developmental; education and learning; clinical and mental health (general); social behaviour; cognition and perception; business psychology and careers; forensic psychology; eating behaviors and body image (specific health sub-theme); other health-related behaviours. Finally, the discussions and conclusions provided by the authors of primary empirical studies were reviewed for their suggested impact on psychological science and/or the local population. 
Fig. 1 Screening process

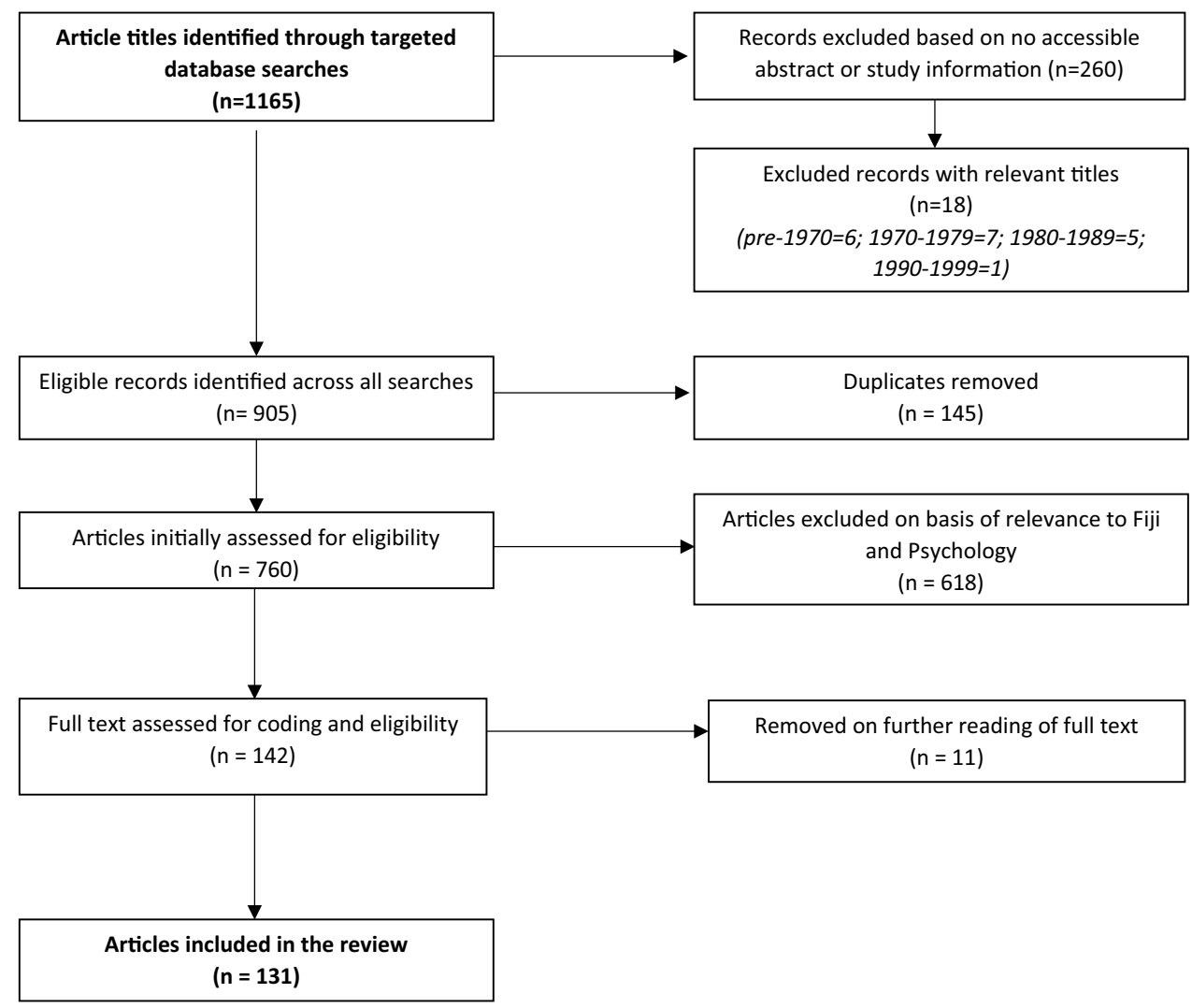

Table 1 Code descriptors for research approach categories

\begin{tabular}{ll}
\hline Code & Descriptor for the purposes of present review \\
\hline Etic & - Studies in which the Fijian sample was part of a multi-country comparison of set phenomena \\
& - Studies in which pre-determined psychological phenomena are being quantitatively assessed and investigated in the \\
& Fijian sample \\
& - Studies in which the aim is to explore and extend known psychological theories from a cross cultural perspective \\
(using Fiji as an opportunity to do this) \\
Emic & - Studies that explore the nature of psychological phenomena within Fijian populations \\
& - Studies that investigate psychological phenomena from a predominantly qualitative or ethnographic perspective \\
- Studies discussing research or practice from a specifically Fijian perspective \\
Indigenous psy- \\
chology
\end{tabular}

\section{Results}

\subsection{Publication year}

There has been a small but consistent increase in studies including a Fijian sample in recent years with $48.1 \%$ of the literature being published in the last 10 years and $25.2 \%$ in the last 5 years (see Fig. 2 ).

\subsection{Article type, methods and samples}

This literature was predominantly primary research publications ( $80.2 \%$ ) of which $42 \%$ were survey-based, $22.9 \%$ used qualitative methods, 9.2\% used experimental procedures (undertaken in field location sites), and 6.1\% analyzed secondary public record data. The remaining publications comprised of literature reviews (6.1\%) and service discussions/ 
Fig. 2 Fiji-based psychological publications by year

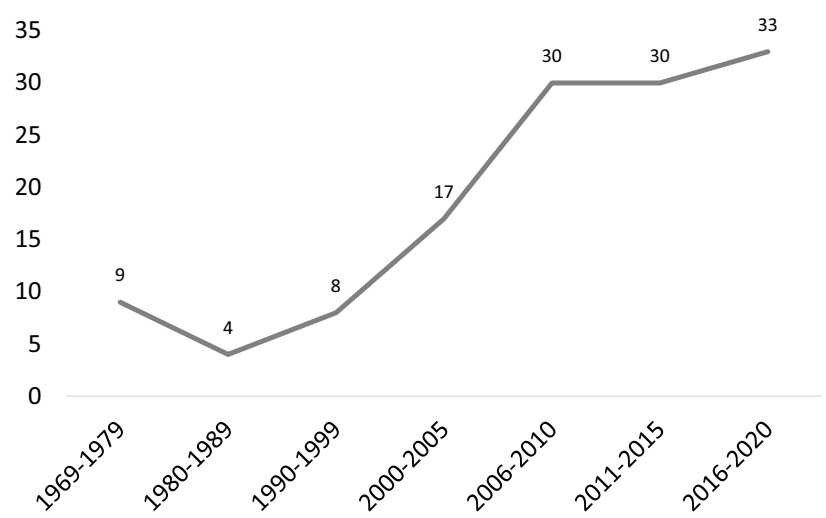

commentaries (13.8\%). The primary data included broad sampling of participants from urban town centres and marketplaces (14.5\%), students in university classes (11.5\%), children and young people in schools in both rural and urban areas (21.4\%), and village populations from rural areas within the main island of Viti Levu (12.2\%) as well as from remote island locations (9.9\%) (8.4\% of primary research articles did not specify location).

\subsection{Cultural approach and purpose of study: etic/emic/indigenous psychology}

The coding for etic, emic and Indigenous approaches indicates $66.92 \%$ (89) of the identified literature would fall under the etic approach and $15.79 \%$ (21) would be categorised as emic research. In addition, $17.29 \%$ (23) would be consistent with a specifically Indigenous psychology perspective (eight of these were categorised as both emic scientific perspective and Indigenous psychology) with the remaining eight articles not being applicable to this categorisation. Of the emic and Indigenous-coded studies, four and eight respectively did not contain any primary empirical data (critical discussion of services, literature reviews and secondary data reports). Also, three of the Indigenous psychology coded articles were discussions of multiple PICs but deemed still to be focused on exploring the nature of indigenous psychology from the regional pacific perspective. Within the etic coded articles, 30 (22.90\% of total) included Fiji data as part of multi-country projects (with 16 of these not providing any specific discussion of the Fiji sample).

\section{Discussion}

\subsection{Summary of research themes and findings}

\subsubsection{Clinical and health behaviours (including eating and body image)}

Nearly half (46.6\%) of the total publications related to mental health services $(27,20.1 \%)$ or health related behaviours (34, $26.0 \%)$. Of these publications 45 (34.4\%) were empirical papers using qualitative $(18,13.7 \%)$ or questionnaire methods $(21,16.0 \%)$. The non-empirical publications were mainly commentaries on mental health services or training workshops (12) or reported either prevalence data on mental health related cases from public service records (6).

A significant number of the empirical articles $(23,17.6 \%)$ related to eating behaviours (including body image and eating disorders) and appeared to stem from the same large-scale, international projects exploring eating disorders and body image perceptions in Fijian youth compared to findings from other countries. Indeed, Becker [29] argued that Fiji specifically provides an opportunity to study changes in eating, health, and body image in a population with both a historic and current exposure to western culture alongside a strong Indigenous identity. For example, McCabe et al. [30] showed that while Fiji adolescents had a high BMI they also had a relatively high body satisfaction compared to other Low and Middle-Income countries that may stem from traditional values and beauty standards. Specifically, Indigenous Fijian ethnic groups believed larger bodies to be well respected, but this was not seen in Indo-Fijian ethnic groups [31]. Also, body image and appearance beliefs were more influenced by parents in Fijian ethnic groups and less by media when compared to Australian adolescents [32, 33]. However, both Becker et al. [29], and Gerbasi et al. [34] also showed 
an influence of social networking and peer influence on Fijian children's eating pathologies despite a lack of affiliation with 'western ideals' in general.

On a separate theme, two articles by O'Connor et al. $[35,36]$ aimed to explore the meaning and understanding of sexual health and behaviours among Fijian youth. The findings show expected socio-cultural influences as well as influence of western ideals and increased individuality. The findings around emotions that drive and block sexual behaviours have important implications for public health policy —not just in Fiji but globally where young people are developing reproductive values and sexual wellbeing in the context of traditional family values and exposure to western ideals.

In contrast to the literature on eating and health behaviours, the mental health literature from Fiji has been restricted to descriptive commentaries of mental health services, or studies specific to the issue of rising suicide rates in Fiji [37-42]. These used secondary data reports from hospital admissions and police data on recorded deaths to describe suicide trends across demographic variables to draw conclusions about risk and protective factors. This lack of research on suicidal ideation is disappointing given the relatively high rates of suicide generally reported across the Pacific Island Countries and in particular among Indo-Fijians within Fiji [41].

Among other publications reporting primary research on mental health specific to Fiji, Aghanwa [43] showed how negative attitudes about the causes of mental illness explained much of the cultural stigma. Taylor et al. [44] present a case study of people affected by a protracted hostage crisis as part of the political coup in Fiji in 2000. The findings highlight the importance of religious belief in adaptation and coping processes during the crisis. It also describes small challenging behaviors undertaken by some of the older women hostages that provided a sense of control through relationship building with the captors (e.g., demanding the return of handbags, starting conversations with younger guards).

\subsubsection{Cognition and social behavior}

Of the identified literature, $29.0 \%$ related to cognition (9.9\%) or social behaviour (19.1\%). The majority of the cognition themes related to religious and cultural influences on attitudes and beliefs. For example, Willard and McNamara [45] found Fijians' conceptualisation of the human mind, the agentic self and the nature of God was more socio-centric and multivariate than North American samples, despite similar Christian religious teachings about God. This finding indicate the collectivist cultural influence not just on social behaviours and values but also on central aspects of one's consciousness and sense of agency. It also shows how consideration of the impact of religion and faith on psychological constructs should also be interpreted within the cultural rather than simple religious denomination context.

Other studies have been able to tease apart the aspects of constructs that are universal versus culturally specified. For example, Tracy et al. [46] compared implicit and explicit responses to emotional expressions between the US and rural Fijian samples. They found that while explicit responses followed predicted lines where happiness is seen as higher status than pride, implicit responses showed cultural differentiation in that Fijians did not seem to differentiate between positive emotions implicitly in the way US students did. This study also begins to indicate the importance of methodology when investigating psychological constructs across different populations.

The review identified a large number of articles exploring prosocial variables within rural communities in Fiji. For example, McNamara and Henrich $[47,48]$ utilized the high communal values, shared resources, and high religious faith of Fijian island small-village populations to explore social bonds and kindship. These studies provided an in-depth understanding of successful communal kinship systems and how cooperative behaviours can extend beyond genetic ties to develop more complex but successful social groups. In a related paper McNamara et al. [49] also showed the greater importance of religious based punishments and concerns in influencing favouritism and resource sharing than police and legal penalties. Indigenous cultural findings such as this add to the broader understanding of how intersectionality of group identities interact in cognitive processes. Similarly, Willard [50] examined group biases and prosocial behaviour among different religious groups in Fiji. In the same population as the McNamara and Henrich studies, Kline et al. [51] report that Fijians, but not Peruvian Indigenous samples, show irrelevant action imitation of White experimenters suggesting that the political context and attitude towards colonization by White Europeans may impact basic social cognitive processes.

Finally, two recent articles considered prejudice and stereotypes in Fijian populations. For example, Johnson et al. [52] investigated the role of trait emotional intelligence as mediating the effect of negative feedback on ethnic group biasthis study was one of the first to apply experimental methods from similar studies in the US to ethnic group tensions in the Pacific (between the Indo-Fijians and the iTaukei, Indigenous Fijian people). Similarly, Johnson et al. [53] assessed the role of media violence (music videos) on perceptions of inter-partner violence among Fijian women. These studies both add to an otherwise largely western literature on these themes, while providing important exploration related to social 
issues prevalent in the Pacific region. This is in line with Adair's [1] discussion of internationalising psychology in a valuable way: where research in developing nations should have both scientific and practical benefit for the local community.

\subsubsection{Child development and learning}

Six studies covered child and developmental themes. Some utilised samples from Fiji and other PICs to explore the impact of culture and environment on children's learning and development. For example, Broesch et al. [54] conducted a study including Indigenous pacific samples from Fiji on children's self-recognition found that few non-western children showed self-recognition in the standard mirror rouge test and none of the Fijian children did. The authors discuss these strong cultural differences in what is often considered a universal cognitive process (self-recognition) in terms of parenting styles and the nature of self-oriented behaviors in strong communal societies as well as the reduced use and meaning of mirrors in non-western cultures. The findings also question the validity of the mirror rouge test itself as a reliable measure of self-cognition and suggest that perhaps, it is a measure of individualistic 'western' notions of self-consciousness.

Similarly, Aime et al. [55] investigated gaze and attention in infants during a caregiver interaction to compare children in urban areas of the US with those in a small rural island of Fiji. They found significantly less motor and attentional activity in the Fijian infants despite no significant differences in caregiver behaviors. The authors suggest such differences may be due to cultural and environmental factors. For example, the rural Fijian infants live in a natural environment with few manufactured toys or electronics compared to the urban infants in the study. This 'simpler' sensory stimulation may lead to differences in attention and activity during interactions, which perhaps, in turn, forms the basis of (or adds to) the cultural characteristic of a 'calm demeanor' in the South Pacific.

Finally, House [56] compared young children from urban and rural areas in Fiji to children from urban Los Angeles (USA) on prosocial behavior in a friendship dyad. The children were given the choice to self-maximise or share a candy reward over a series of high and low-cost trials (within the same dyad). The results showed a similar development of prosocial behaviour by age seven in all samples. However, the level of cooperation and reciprocity differed as a product of overall culture and not as a product of rural or urban living. The authors note this is of interest because it suggests an interplay between universal developmental adaptive strategies (resource sharing develops as an adaptive social strategy) and cultural characteristics and beliefs (resource sharing was predicted more by nationality than social ecology) [see also 57].

\subsubsection{Other applied psychology themes}

Overall, $19.8 \%$ of the publications were on other applied fields of education, forensic and business psychology: 26 (12.2\%) related to education; eight (6.1\%) related to business organisations; and two (1.5\%) forensic psychology. Within this theme, Van Gelder et al. [58] showed that successful entrepreneurs had significantly different goal setting and planning strategies compared to unsuccessful small business owners. Small, developing economies such as Fiji can provide a fertile setting for investigations of the psychology behind bottom-up entrepreneurship, small and family run business leadership.

Similarly, De Vries et al. [59] investigated the personality and environmental pathways leading to unethical business decisions within a Fijian sample. They found that people higher on humility-honesty turn away from unethical but rewarding opportunities not just because of a cognitive value appraisal but also because it produces a negative emotional response in them. The authors argue that by undertaking the research in Fiji they were able to test theories in a specifically non-western setting and one with less regulation and higher perceived corruption. In a way this allows for a more authentic assessment of the individual psychological constructs involved in decision making.

In the field of education and learning, Phan [60-64] conducted a series of studies in a Fijian University on students reflective thinking, epistemological beliefs, and approaches to learning. These studies are important to explore the impact of Indigenous cultural traits on expected aspects of 'western' models of higher education such as critical and reflective thinking, deep learning, and independent motivation. Phan $[60,61]$ found that supporting the academic self-efficacy in Indigenous Pacific students can have a direct impact on reflective thinking and as a result enhances deep learning and academic performance. Additionally, these studies suggested the type of epistemological beliefs held by a student associated with their motivation goals, self-regulation, and approach to learning [62-64]. Again, this is important as beliefs about the structure and development of knowledge are culturally informed but could be addressed and developed as part of higher education curricula. These applied studies add to both the theoretical knowledge as well as the indigenous psychology perspective as they could be used in teacher training and educational strategies locally (although it is unclear if this application was an overt intention of the research). 
Finally, as climate change becomes a significant global topic in all research fields, small island nations such as Fiji are at the center of understanding the psychological effects of environmental change and climate disasters. In the literature reviewed, only two articles related to climate related themes: DuBray et al. [65] who showed Fijians expressed sadness rather than anger about climate threats when compared to other groups; and Fairley et al. [66] who presented a case study on trauma and resilience in a community impacted by cyclones.

\subsection{Fiji research and the globalisation of psychology}

As described above the ability to investigate particular environmental and socio-cultural variables found in Fijian populations has impacted psychological understanding in several ways. For example, authors have discussed their findings in terms of:

- the influence of communal social structures and parenting styles on self-related cognitive processes (e.g., self-recognition):

- the role of natural rural environments as opposed to manufactured environments on children's attention activity;

- the influence of sociocultural factors on the conceptualisation of religious teachings

- the expanded nature of kinship social cognitive processes as a necessary element of social cohesion in collectivist communities

- the historical nature and outcomes of political colonisation as impacting social cognitive processes between Indigenous groups and those representing historic colonisation (i.e. white Europeans).

In addition, findings from applied themes within Fijian samples, particularly in the fields of health and mental health, have shown the subtle interplay between traditional cultural factors and media exposure on body image, eating behaviors and wellbeing. This is likely to be reflected in many other countries where exposure to western social media becomes more ubiquitous among adolescent populations.

One particular opportunity provided by the Fijian research context is exploring the differences between the rural, island community samples and the urban samples. That is, the benefit of Pacific Island nations such as Fiji, where communities are widely spread across different islands is the vast and continuing diversity in lifestyle and western media exposure and between urban and rural populations. This may not be seen in studies of Indigenous populations within more geographically connected countries. Fessler et al. [67] discuss the importance of including samples from varying population scales, values, modes of subsistence and exposure to mass media Fiji allows exploration of these variables while also providing an underlying cultural diversity widely different from comparisons in western and developed nations. Furthermore, the geographic and lifestyle diversity of small island nations such as Fiji provide a critically important context for investigating the psychological impact of global issues such as climate change. While this is yet to be explored in any depth (compared to other identified themes), Fiji populations provide an opportunity to explore climate change within a single country and culture but where environmental traditions, exposure, and dependency differ widely.

One aim of the present review was to analyse the extent to which the Fiji research reflects globalisation in terms of etic, emic and Indigenous psychology approaches. On the one hand, both etic and emic approaches have been employed within the identified literature which is a positive indicator of building a comprehensive globalisation in research aims and outcomes. However, it is still the case that the most common form of research undertaken in Fiji has been etic style studies in which the Fijian sample is used as a cross-cultural comparator for western-driven variables and research questions and in project funded and led by non-Fiji based primary authors. This is also indicated by the number of studies (19.1\%) where a Fiji sample was used as part of a multi-country project and with little or no specific comment on Fiji itself. This predominantly etic form of cultural globalisation reflects the issue discussed by Berry [4] about cross-cultural comparative research inadvertently resulting in a form of exploitation of Indigenous cultures rather than being open to an Indigenous context for developing psychological research questions.

One particular consideration from a predominantly etic literature base is the appropriateness of the methods themselves applied in Indigenous Pacific contexts. From the current review, the Fijian literature identified from international publication databases predominantly utilised standard questionnaire, interview, or experimental methods, as are common in psychological research. It is important to consider how these tools are used in Indigenous populations and especially where there is a diversity in language, literacy, and understanding of paper/pencil assessments. Several of the studies mention using a verbal interview technique for questionnaires through a local interviewer; this is important 
in cultures where there is a strong sense of community and protocols for gathering information about a village sample. However, it is not clear how this may impact responses and the potential power dynamics revolving the ethical principles of voluntary consent and the right to withdraw. In particular, research undertaken in remote village communities would need to respect the local customs of Matagali (a communal, land-owning tribal group) and Village Chiefs, and this could potentially mean that where permission is gained from a chief, individual villagers feel obligated to participate.

Beyond practical considerations for translating methods into a local context, some Indigenous authors have discussed the importance of considering Pacific worldviews as a basis for developing the research methodologies themselves [68]. In her article, Ponton [68] describes different approaches to ethnographic and social science research developed from Pacific beliefs and traditions that may lead to more valid and ethical data. For example, the Fonofale model of wellbeing [69] has been used as an approach to evaluating mental health services and outcomes in Pacific populations and as a tool for developing research procedures (such as participant sampling and recruitment) that are more culturally sensitive enriching the research outcomes. In addition, the Indigenous community forum called Talanoa may be an important structure for qualitative research (as opposed to standard focus group or interview methods) [70]. That is, through the inclusion of traditional forum processes, which focus on rapport and relationships, Pacific participants may feel more respected and therefore more trusting of the research process. While these discussions have led to some incorporation of these Indigenous worldviews within the Pacific mental health sector [71], they are yet to be integrated into psychological research more broadly, at least as identifiable from the international literature base.

A second aspect of the Indigenous psychology research field is that it should serve both a scientific and local social or development purpose in such smaller, lower income nations. To some extent, this was partially fulfilled by the series of publications investigating eating behaviors and body image in Fiji and the Pacific in light of the need to address important public health concerns such as high obesity and non-communicable disease prevalence in the Pacific region. Yet, there are a range of other social and psychological issues also known to be prevalent in the region, that are little represented in the published psychological literature. For example, the absence of publications investigating variables related to mental health and wellbeing in Fijian psychology (as opposed to practice and service reviews). Koc and Kafa [8] in their review on cross cultural research in psychotherapy specifically point out the need to assess psychotherapy models in underdeveloped cultures as critical in developing valid and sustainable mental health support services. Moreover, there are elements of the Fiji and Pacific cultural context that would provide important opportunity to understand mental health, symptom presentation, help seeking and stigma from a more global perspective.

Furthermore, the articles relating to themes in social conflict, gender and health-related behaviours were seemingly individual studies without any follow-up. For example, individual studies investigating sexual health and wellbeing [35, 36] ethnic group conflict [52], media and inter-partner violence [53] and attitudes towards mental health issues such as depression [72], disability [73] and Dementia [43, 74] seem to stand alone despite significant findings. This pattern of single-study publications suggests that at least in terms of socially impacting Indigenous research there needs to be greater effort put into building ongoing and sustainable research projects.

\section{Conclusions and future directions}

The discussion indicates that the Pacific Island nation of Fiji has the infrastructure to support psychological research of an international standard and that Indigenous populations across the Fijian islands are open to participating in primary research using a range of methods. As a result, Fijian-based research has already added to established psychological theory in a number of key areas and applied areas of research such as climate change or trauma and resilience processes could potentially provide valuable scope for future studies. Importantly, alongside more traditional etic approaches and cross-cultural comparisons, research should be targeted that takes a more emic approach to explore psychological constructs unique to Fijian or Pacific Island populations. Several models of Indigenous methods have begun to be explored within wellbeing and health disciplines that could inform and extend psychological science in future research. Finally, this review would argue that the globalisation of psychological science must come not only from funding individual studies, but from investing in sustainable programmes of research allowing for the development of findings and investigations over time. Indeed, partnerships between international academic institutions and Pacific Island Countries could provide long term benefits to maintaining a global perspective in psychological science and ensuring psychological research serves a positive role for global communities, particularly in LMICs such as Fiji. 
Acknowledgements Special thanks to Leslie Aguilar for assistance in developing a database of articles for the literature review and Sierra Meyer for assistance with formatting and copyediting.

Authors' contributions AEC and MAW were involved in the research design and analysis. AEC wrote the first draft of the manuscript, with subsequent drafts being reviewed and edited by both AEC and MAW. Both authors read and approved the final manuscript.

Funding None.

\section{Declarations}

Competing interests The authors declare no competing interests.

Open Access This article is licensed under a Creative Commons Attribution 4.0 International License, which permits use, sharing, adaptation, distribution and reproduction in any medium or format, as long as you give appropriate credit to the original author(s) and the source, provide a link to the Creative Commons licence, and indicate if changes were made. The images or other third party material in this article are included in the article's Creative Commons licence, unless indicated otherwise in a credit line to the material. If material is not included in the article's Creative Commons licence and your intended use is not permitted by statutory regulation or exceeds the permitted use, you will need to obtain permission directly from the copyright holder. To view a copy of this licence, visit http://creativecommons.org/licenses/by/4.0/.

\section{References}

1. Adair JG, Coelho AEL, Luna JR. How international is psychology. Int J Psychol. 2002;37(3):160-70.

2. Arnett JJ. The neglected 95\%: why American psychology needs to become less American. Am Psychol. 2008;63(7):602-14.

3. Bauserman R. International representation in the psychological literature. Int J Psychol. 1997;32(2):107-12.

4. Berry JW. Global psychology. S Afr J Psychol. 2013;43(4):391-401.

5. Henrich J, Heine SJ, Norenzayan A. Most people are not WEIRD. Nature. 2010;466:29.

6. Tindle R. Improving the global reach of psychological research. Discov Psychol. 2021;1:5. https://doi.org/10.1007/s44202-021-00004-4.

7. Echo-Hawk HMS. Indigenous communities and evidence building. J Psychoact Drugs. 2011;43(4):269-75.

8. Koc V, Kafa G. Cross cultural research on psychotherapy: the need for a change. J Cross Cult Psychol. 2019;50(1):100-15.

9. Thomas T. Psychology in a culturally diverse society. Aust Psychol. 2004;39(2):103-6.

10. McNamara RA, Naepi S. Decolonising community psychology by supporting indigenous knowledge, projects and students: lessons from Aotearoa New Zealand. Am J Community Psychol. 2018;62:340-9.

11. Webster GD, Nichols AL, Schember TO. American psychology is becoming more international. Am Psychol. 2009;64(6):566-8.

12. Apicella CL, Norenzayan A, Henrich J. Beyond WEIRD: a review of the last decade and a look ahead to the global laboratory of the future. Evol Hum Behav. 2020;41:319-29.

13. Thalmayer AG, Toscanelli C, Arnett JJ. The neglected 95\% revisited: is American psychology becoming less American? Am Psychol. 2021;76(1):116-29.

14. Bauer PJ. Expanding the reach of psychological science (Editorial). Psychol Sci. 2020;31(1):3-5.

15. Chen SX. From emic to etic: exporting indigenous constructs. Soc Pers Psychol Compass. 2010;4(6):364-78.

16. Rolland JP. Cross-cultural generalizability of the five-factor model of personality. In: McCrae RR, Allik J, editors. The five-factor model of personality across cultures. Boston: Springer Sciences; 2002. p. 7-29.

17. Cheung FM, van de Vijver FJR, Leong FTL. Toward a new approach to the study of personality in culture. Am Psychol. 2011;66(7):593-603.

18. Butaverde V, de Raad B, Zanfirescu A-S. An emic-etic approach to personality assessment in predicting social adaptation, risky social behaviors, status striving and social affirmation. J Res Pers. 2018;76:113-23.

19. Butaverde V, Iliescu D. Emic vs etic frame of reference personality assessment in the prediction of work-related outcomes. Career Dev Int. 2019;24(7):686-701.

20. Adair JG, Diaz-Loving R. Indigenous psychologies: the meaning of the concept and its assessment. Appl Psychol Int Rev. 1999;48(4):397-402.

21. Adair JG, Puhan BN, Vohra N. Indigenisation of psychology: empirical assessment of progress in Indian research. Int J Psychol. 1993;28(2):149-69.

22. Adair JG, Kagitcibasi C. Development of psychology in developing countries: factors facilitating and impeding its progress. Int J Psychol. 1995;30(6):633-41.

23. Chakkarath P. The role of indigenous psychologies in the building of basic cultural psychology. In: Valsiner J, editor. The Oxford handbook of culture and psychology. Oxford: Oxford University Press; 2012. p. 71-95.

24. Poortinga YH. Integration of basic controversies in cross-cultural psychology. Psychol Dev Soc. 2016;28(2):161-82.

25. Khoo S, Morris T. Physical activity and obesity research in the Asia-Pacific: a review. Asia Pac J Public Health. 2012;24(3):435-49.

26. Wang Y, Lim H. The global childhood obesity epidemic and the association between socio-economic status and childhood obesity. Int Rev Psychiatry. 2012;24(3):176-88.

27. Sakundarno M, Bertolatti D, Maycock B, Spickett J, Dhaliwal S. Risk fctors for Leptospirosis infection in humans and oplications for public health in Indonesia and the Asia-Pacific region. Asia Pac J Public Health. 2014;26(1):15-32.

28. Reich AJ, Claunch KD, Verdeja MA, Dungan MT, Anderson S, Clayton CK, Goates MC, Thacker EL. What does "successful ageing" mean to you?-Systematic review and cross cultural comparison of lay perspectives of older adults in 13 countries, 2010-2020. J Cross Cult Gerontol. 2020;35:455-78. 
29. Becker AE, Fay KE, Agnew-Bais J, Khan N, Striegel-moore RH, Gilman SE. Social network media exposure and adolescent eating pathology in Fiji. Br J Psychiatry. 2011;198:43-50.

30. McCabe MP, Fuller-Tyszkiewicz M, Mellor D, Ricciardelli L, Skouteris H, Mussap A. Body satisfaction among adolescents in eight different countries. J Health Psychol. 2011;17(5):693-701.

31. McCabe MP, Waqa G, Dev A, Cama T, Swinburn BA. The role of cultural values and religion on views of body size and eating practices among adolescents from Fiji, Tonga and Australia. Br J Health Psychol. 2013;18:383-94.

32. Williams LK, Ricciardelli LA, McCabe MP, Swinburn BA, Waqa G, Bavarda K. A comparison of the sources and nature of body image messages perceived by Indigenous Fijian and European Australian Adolescent Girls. Sex Roles. 2006;55:555-66.

33. McCabe MP, Mavoa H, Ricciardelli LA, Waqa G, Fotu K, Goundar R. Sociocultural influences on body image among adolescent boys from Fiji, Tonga, and Australia. J Appl Soc Psychol. 2011;41(11):2708-22.

34. Gerbasi ME, Richards LK, Thomas JJ, Agnew-Blais JC, Thompson BH, Gilman SE, Becker AE. Globalisation and eating disorder risk: peer influence, perceived social norms and adolescent disordered eating in Fiji. Int J Eat Disord. 2014;47:727-37.

35. O'Connor M, Rawstone P, Iniakwala D, Razee H. Adolescent emotional wellbeing and sexual reproductive health-seeking behaviors. Sex Res Soc Policy. 2019;16:373-84.

36. O'Connor M, Rawtsone P, Devi R, Iniakwala D, Razee H. Understanding the meaning of youth sexual and reproductive wellbeing in Fiji. Cult Health Sex. 2018;20:1-15.

37. Auxier CR, Forster PM, Kuruleca SC. Counseling in Fiji. J Ment Health Couns. 2005;27(2):142-8.

38. De Leo D, Milner A, Fleischman A, Bertolote J, Collings S, Amadeo S, Chan S, Yip PSF, Huang Y, Saniel B, Lilo F, Lilo C, David AM, Benavente $B$, Nadera D, Pompili M, Kolves KE, Wang X. The WHO START study: suicidal behaviors across different areas of the world. Crisis. 2013;34(3):156-63.

39. Devaney ML, Reid G, Baldwin S, Crofts N, Power R. Illicit drug use and responses in six Pacific Island countries. Drug Alcohol Rev. 2006;25:387-90.

40. Forster PM, Kuruleca SC, Auxier CR. A note on the recent trends in suicide in Fiji. J Pac Rim Psychol. 2007;1 (1):1-4.

41. Mathieu S, de Leo D, Koo YW, Leske S, Goodfellow B, Kolves K. Suicide and suicide attempts in the Pacific Islands: a systematic literature review. Lancet Reg Health West Pac. 2021;17: 100283.

42. Aghanwa $\mathrm{H}$. The determinants of attempted suicide in a general hospital setting in Fiji Islands: a gender specific study. Gen Hosp Psychiatry. 2004;26:63-9.

43. Aghanwa HS. Attitude toward and knowledge about mental illness in Fiji islands. Int J Soc Psychiatry. 2004;50(4):361-75.

44. Taylor AJW, Nailatikau E, Walkey FH. A hostage Trauma assignment in Fiji. Australas J Disaster Trauma Stud. 2002;2002(2).

45. Willard AK, McNamara RA. The minds of Gods and Humans: differences in mind perception in Fiji and North America. Cogn Sci. 2019;43: e12703.

46. Tracy JL, Zhao W, Shariff AF, Henrich J. Cross cultural evidence that the nonverbal expression of pride is an automatic status signal. J Exp Psychol Gen. 2013;42(1):163-80.

47. McNamara RA, Henrich J. Kin and kinship psychology both influence cooperative coordination in Yasawa Fiji. Evol Hum Behav. 2017;38:197-207.

48. McNamara RA, Henrich J. Jesus vs the ancestors: how specific religious beliefs shape prosociality on Yasawa Island, Fiji. Relig Brain Behav. 2018;8(2):185-204.

49. McNamara RA, Norenzayan A, Henrich J. Supernatural punishment, in group biases and maternal insecurity: experiments and ethnography from Yasawa, Fiji. Relig Brain Behav. 2016;6(1):34-55.

50. Willard AK. Religion and prosocial behavior among the Indo-Fijians. Relig Brain Behav. 2018;8(2):227-42.

51. Kline MA, Gervais MM, Moya C, Boyd RT. Irrelevant action imitation is short term and contextual: evidence from two under studied populations. Dev Sci. 2020;23: e12903.

52. Johnson J, Lakhan R, Lecci L, Dovidio JF, Schellhaas FMH. Trait emotional intelligence moderates the impact of failure feedback: out group derogation in Fiji. Soc Psychol Personal Sci. 2020;11(7):975-82.

53. Johnson J, Edwards W, Malamuth M, Lecci L. Feeling my sisters pain: perceived victim suffering moderates the impact of sexualized music videos on Fijian Women's responses to Men's intimate partner violence against women. Sex Roles. 2020;83(7-8):510-22.

54. Broesch T, Callaghan T, Henrich J, Murphy C, Rochat P. Cultural variations in children's mirror self-recognition. J Cross Cult Psychol. 2011;42(6):1018-29.

55. Aime $H$, Rochat $P$, Broesch T. Cultural differences in infant spontaneous behaviour: evidence from a small scale, rural island society. Infant Child Dev. 2020;30: e2204.

56. House BR. Diverse ontogenies of reciprocal and prosocial behaviour: operative development in Fiji and the United States. Dev Sci. 2017;20: e12466.

57. House BR, Silk JB, Henrich J, Barrett C, Scelza BA, Boyette AH, Hewlett BS, McElreath R, Laurence S. Ontogeny of prosocial behvior across diverse societies. PNAS. 2013;110(36):14586-91.

58. Van Gelder J, De Vries RE, Frese M, Goutbeek J. Differences in psychological strategies of failed and operational business owners in the Fiji Islands. J Small Bus Manag. 2007;45(3):388-400.

59. De Vries RE, Pathak RD, Van Gelder J, Singh G. Explaining unethical business decisions: the role of personality, environment and states. Personal Individ Differ. 2017;117:188-97.

60. Phan HP. An examination of reflective thinking, learning approaches and self-efficacy beliefs at the University of the South Pacific: a path analysis approach. Educ Psychol. 2007;27(6):789-806.

61. Phan HP. Exploring students reflective thinking practice, deep processing strategies, effort, and achievement goal orientations. Educ Psychol. 2009;29(3):297-313.

62. Phan HP. Exploring epistemiological beliefs and learning approaches in context: a sociocultural perspective. Electron J Res Educ Psychol. 2008;6(3):793-822.

63. Phan HP. Multiple regression analysis of epistemiological beliefs, learning approaches and self-regulated learning. Electron J Res Educ Psychol. 2008;6(1):157-84. 
64. Phan HP. Predicting change in epistemiological beliefs, reflective thinking and learning styles: a longitudinal study. Br J Educ Psychol. 2008;78:75-93.

65. Du Bray M, Wutich A, Larson KL, White DD, Brewis A. Anger and sadness: gendered emotional responses to climate threats in four island nations. Cross Cult Res. 2019;53(1):58-86.

66. Fairley M, Langeluddecke P, Tennant C. Psychological and physical morbidity in the aftermath of a cyclone. Psychol Med. 1986;16(3):671-6.

67. Fessler DMT, Holbrook C, Gervais MM. Men's physical strength moderates conceptualisations of prospective foes in two disparate societies. Hum Nat. 2014;25:393-409.

68. Ponton V. Utilizing Pacific methodologies as inclusive practice. SAGE Open. 2018. https://doi.org/10.1177/2158244018792962.

69. Pulotu-Endemann FK. Fonofale model of health. 2009. https://www.springboardtrust.org.nz/news/a-resilient-home-the-fonof ale-model-of-health.

70. Vaioleti TM. Talanoa research methodology: a developing position on Pacific research. Waikato J Educ. 2006;12(1):21-34.

71. Ioane J, Tudor K. The Fa'asamoa, person-centered theory and cross-cultural practice. Person-Centered Exp Psychother. 2017;16(4):287-302.

72. Ho GWK, Bressington D, Leung SF, Lam KKC, Leung AYM, Molassiotis A, Ligot J, Ranoco C, Sophal C, Valimaki M. Depression literacy and health seeking attitudes in the Western Pacific region: a mixed methods study. Soc Psychiatry Psychiatr Epidemiol. 2018;53:1039-49.

73. Hopf SC, McLeod S. Services for people with communication disability in Fiji: barriers and drivers for change. Rural Remote Health. $2015 ; 15: 2863$.

74. Leung AYM, Leung SF, Ho GWK, Bressington D, Molassiotis A, Lam C, Ligot J, Valdez LP, Lazalita LA, Chhit S, Chou P, Valimaki M. Dementia literacy in Western Pacific countries: a mixed methods study. Int J Geriatr Psychiatry. 2019;34:1815-25.

Publisher's Note Springer Nature remains neutral with regard to jurisdictional claims in published maps and institutional affiliations. 